\title{
Clinico-hematological Analysis of Pancytopenia in Adults - A Two Year Prospective Study
}

\author{
Reenal R Patel, Manisha Tambekar* and Reeta Dhar \\ Department of Pathology, MGM Medical College and Hospital, Mumbai, Maharashtra, India
}

\begin{abstract}
Background: Pancytopenia is a common hematological finding resulting from various diseases which requires thorough clinical history, physical examination and blood investigations so as to evaluate the cause and plan the management of pancytopenic patients. The aim of this study is to determine the incidence, various causes, common clinical presentations of pancytopenia and to correlate hematological parameters with clinical findings in differentiating causes of pancytopenia.

Methods: It was a two year prospective study which included patients of age 15 years and above having pancytopenia on blood film examination $(\mathrm{Hb}<10 \mathrm{~g} / \mathrm{dl}, \mathrm{TLC}<4000 / \mathrm{cmm}$ and platelets $<1 \mathrm{lakh} / \mathrm{cmm})$. Total 226 cases of pancytopenia were evaluated clinically along with hematological parameters and bone marrow aspiration in Central Laboratory of Department of Pathology, Tertiary care hospital, Navi Mumbai.

Results: The most common causes of pancytopenia were malaria (50\%), megaloblastic anemia (18.6\%) and dengue (18.1\%) followed by hypersplenism(7.1\%) \& iron deficiency anemia(2.7\%). The other uncommon causes were septicemia (2.2\%), AIDS(1.8\%), tuberculosis (1.8\%), aplastic anemia $(1.3 \%)$, leptospirosis $(0.9 \%)$, dimorphic anemia $(0.9 \%)$, dyskeratosis congenita $(0.4 \%)$ and myelodysplastic syndrome $(0.4 \%)$. The age of the patients ranged from $15-85$ years with male preponderance. The most common clinical features were weakness $(80 \%)$, fever $(72.1 \%)$, pallor $(100 \%)$ and hepatomegaly $(62.8 \%)$. Normocytic normochromic was the predominant blood picture.
\end{abstract}

Conclusion: The present study concludes that detailed primary hematological investigations and other supportive biochemical investigations can be helpful to rule out or to diagnose causes of pancytopenia and invasive procedures like bone marrow aspiration or biopsy can be avoided in majority of cases.

Keywords: Pancytopenia, Malaria, Dengue, Megaloblastic Anemia.

\section{Introduction}

Pancytopenia is a disorder in which all three major formed elements of blood (red blood cells, white blood cells and platelets) are decreased than normal. It is a common clinical problem with an extensive differential diagnosis. The pattern of diseases leading to pancytopenia may vary in different population groups with their differences in age, nutritional status and prevalence of infection. ${ }^{[1]}$ Thorough physical examination, clinical history and peripheral blood picture can provide valuable information in diagnosing pancytopenia. Studies done in India stress the importance of megaloblastic anemia followed by aplastic anemia as being the major causes of pancytopenia. ${ }^{[2],[3]}$

\section{Material and Methods}

A prospective study was carried out over a period of 2 years in MGM Medical College and Hospital, Kamothe, Navi Mumbai. Ethical committee approval was obtained prior to the commencement of the study.

Patients $\geq 15 y$ rs and both genders were included. Clinical history was obtained along with hematological investigations and peripheral smear examination was performed. A total of 226 cases were selected based on the criteria: Haemoglobin: $<10 \mathrm{gm} / \mathrm{dl}$, total leucocyte count: $<4000 / \mathrm{cmm}$ and platelet count: $<$ one lakh/cmm. ${ }^{[4],[5],[6],[7],[8]}$ Patients on chemotherapy or radiotherapy, diagnosed cases of pancytopenia and recently received blood transfusions were excluded from this study.

EDTA anticoagulated blood $(2 \mathrm{ml})$ was collected, processed through automated 5part differential cell analyzer and hematological parameters were obtained. Peripheral blood smear was stained with Field's and Leishman stain and examined for RBC, WBC and platelet morphology along with hemoparasites. Anemias were classified morphologically as normocytic normochromic, microcytic hypochromic, macrocytic \& dimorphic anemia. Informed consent was taken before performing bone marrow aspiration in cases where it was required. Special tests: Rapid malarial antigen test, dengue card test, leptospirosis card test, tests for HIV, Hepatitis viruses, serum vitamin B12, serum iron studies, liver and renal function tests and tests for tuberculosis, USG abdomen, CT and MRI scans, PET scans, etc. were also performed. 


\section{Results}

The age of the patients ranged from 15-85 years. The commonest age group affected was 15-25years (30.5\%) followed by $26-35 y e a r s ~(22.6 \%), 36-45 y e a r s ~(16.4 \%)$ and 46-55years (11\%). 137 were males and 89 were females (M:F ratio $1.5: 1)$.

The commonest mode of presentation was weakness $(80.1 \%)$, fever $(72.1 \%)$, dyspnoea $(23 \%)$ and bleeding tendency $(11.5 \%)$. Pallor (100\%) was the most common clinical sign followed by hepatomegaly (62.8\%), splenomegaly (47.8\%) and lymphadenopathy (4.9\%). Out of 226 cases of pancytopenia, 18 cases had mixed etiology hence the total is 244 . We graded pancytopenia as mild, moderate and severe. We observed moderate anemia (70.4\%), mild leucopenia $(69.9 \%)$ and mild thrombocytopenia $(56.2 \%)$ with predominant normocytic normochromic blood picture. The most common cause was malaria $(50 \%)$ followed by megaloblastic anemia $(18.6 \%)$ and dengue $(18.1 \%)$.

Table 1: Incidence of various causes of pancytopenia.

\begin{tabular}{|l|c|c|}
\hline Causes & Number of Cases & Percentage ( \%) \\
\hline Malaria & 113 & 18.6 \\
\hline Megaloblastic anemia & 42 & 18.1 \\
\hline Dengue & 41 & 7.1 \\
\hline Hypersplenism & 16 & 2.7 \\
\hline Iron deficiency anemia & 06 & 2.2 \\
\hline Septicemia & 05 & 1.8 \\
\hline AIDS & 04 & 1.8 \\
\hline Tuberculosis & 04 & 1.3 \\
\hline Aplastic anemia & 03 & 0.9 \\
\hline Leptospirosis & 02 & 0.9 \\
\hline Dimorphic anemia & 02 & 0.4 \\
\hline Dyskeratosis congenita & 01 & 0.4 \\
\hline Myelodysplastic syndrome & 01 & 1.8 \\
\hline Undiagnosed & 04 & \\
\hline Total & $244^{*}$ & \\
\hline
\end{tabular}

*Out of total 226 cases of pancytopenia, 18 cases had mixed etiology hence the total is 244 .

Table 2: Comparison of causes of pancytopenia in various studies.

\begin{tabular}{|c|c|c|c|c|c|c|c|}
\hline Study & Country & Year & $\begin{array}{c}\text { Number of } \\
\text { cases }\end{array}$ & $\begin{array}{c}\text { Age } \\
\text { Group } \\
\text { (years) }\end{array}$ & $\begin{array}{l}\text { Common } \\
\text { cause }\end{array}$ & $\begin{array}{l}\text { Second } \\
\text { cause }\end{array}$ & $\begin{array}{l}\text { Third } \\
\text { cause }\end{array}$ \\
\hline Sweta et al. ${ }^{[14]}$ & India & 2014 & 100 & $5-80$ & MA & $\mathrm{AA}$ & $\mathrm{ML}$ \\
\hline Tonape et al. ${ }^{[4]}$ & India & 2014 & 210 & All & MA & AA & ALL \\
\hline Dahake et al. ${ }^{[9]}$ & India & 2014 & 94 & All & MA & $\mathrm{AA}$ & $\mathrm{AL}$ \\
\hline Soma et al. ${ }^{[5]}$ & India & 2013 & 60 & All & $\mathrm{AA}$ & MA & $\mathrm{AL}$ \\
\hline Parmar et al. ${ }^{[19]}$ & India & 2013 & 100 & $1-95$ & MA & $\mathrm{ML}$ & AA \\
\hline Thakkar et al.[11] & India & 2013 & 100 & $>12$ & MA & $\mathrm{ML}$ & $\mathrm{HS}$ \\
\hline Jain et al. ${ }^{[10]}$ & India & 2013 & 250 & All & $\mathrm{HS}$ & IF & MS \\
\hline Tareen et al. ${ }^{[18]}$ & Pakistan & 2012 & 180 & All & $\mathrm{ML}$ & LK & TB \\
\hline Gayathri et al. ${ }^{[16]}$ & India & 2011 & 104 & $2-80$ & MA & $\mathrm{AA}$ & SLL \\
\hline Hamid et al. ${ }^{[12]}$ & Yemen & 2008 & 75 & $3-85$ & $\mathrm{ML}$ & $\mathrm{HS}$ & MA \\
\hline Niazi et al. ${ }^{[7]}$ & Pakistan & 2004 & 89 & $1-30$ & BM Aplasia & MA & $\mathrm{HS}$ \\
\hline Khunger et al. ${ }^{[8]}$ & India & 2002 & 200 & $2-70$ & MA & $\mathrm{AA}$ & SLL \\
\hline Tilak et al. ${ }^{[17]}$ & India & 1999 & 77 & $5-70$ & MA & AA & $\mathrm{ML}$ \\
\hline Our Study & India & 2015 & 226 & $\geq 15$ & $\mathrm{ML}$ & MA & Dengue \\
\hline
\end{tabular}

AA: Aplastic anemia, AL: Acute Leukemia, ALL: Acute Lymphoblastic Leukemia, AML: Acute Myeloid Leukemia, BM Aplasia: Bone Marrow Aplasia, HS: Hypersplenism, IF: Infections, LK: Leukemia MA: Megaloblastic Malaria, ML: Malaria, MS: Myelodysplastic syndrome, SLL: Subleukemic Leukemia, TB: Tuberculosis. 


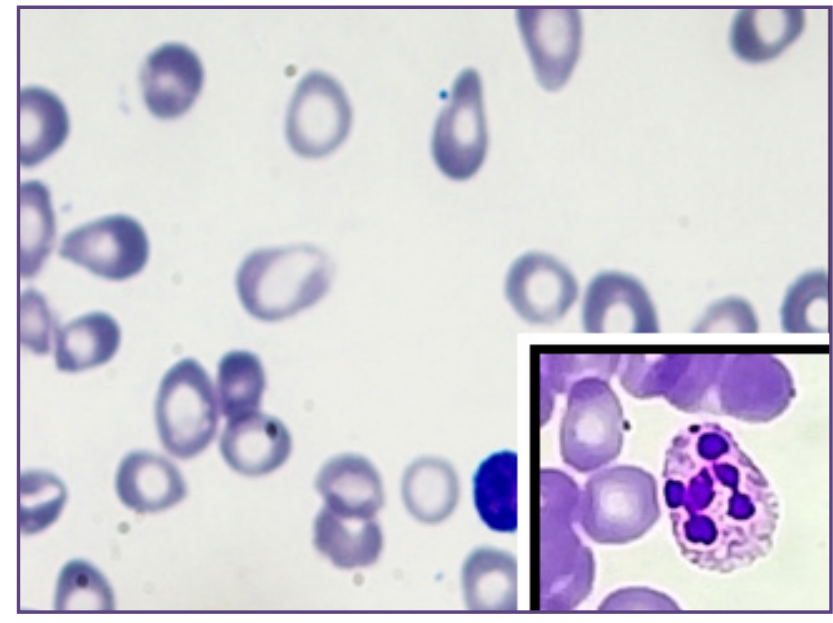

Fig. 1: Macrocytic anemia- Macroovalocytes with marked degree of anisopoikilocytosis, tear drop cells and HowellJolly body. Inset shows a hypersegmented neutrophil. (Field's stain, 1000x).

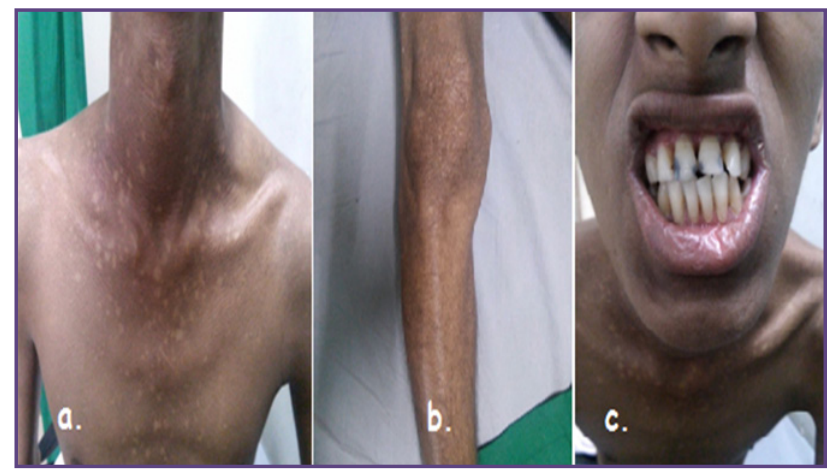

Fig. 2: Dyskeratosis congenita- a,b. Reticular hypopigmentation and hyperpigmentation on neck, chest and leg; c. Dental caries and plaque accumulation.

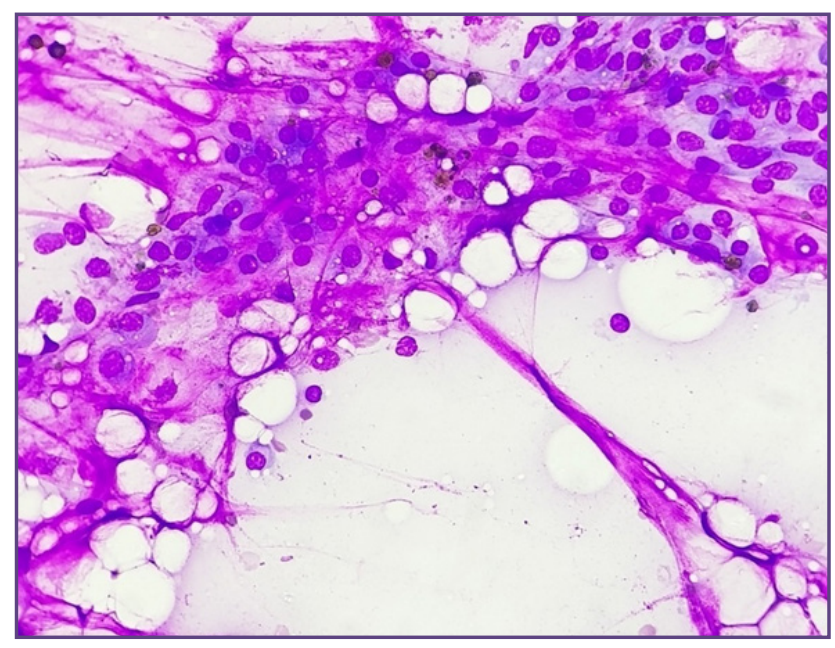

Fig. 3: Dyskeratosis congenita- BM aspirate smear showing hypoplastic marrow with increase in lymphocytes and plasma cells (Leishman's stain, 1000x)

\section{Discussion}

Pancytopenia is a common haematological finding where red blood cells, white blood cells and platelets are decreased below their normal lower limit leading to simultaneous presence of anemia, leucopenia and thrombocytopenia.

Pancytopenia develops mainly by 1) Decrease in hematopoietic cell production as a result of replacement by abnormal or malignant cells, B12 or folate deficiency, destruction of marrow tissue by toxins/drugs, 2) Sequestration ofhematopoietic cells, e.g., hypersplenismand 3) Increased destruction, e.g., sepsis, immune-mediated. ${ }^{[9]}$

The age varied from 15-85 years and the commonest age group affected was $15-25$ years which was similar to the findings observed by Anita et al ${ }^{1}$ followed by 26-35 years. Jain et $\mathrm{al}^{10}$, observed $31-40$ years of age group being the commonest followed by 21-30 years of age group. There were 137 males and 89 females with male: female ratio being 1.5:1. A male predominance has been reported by many authors in their studies. ${ }^{[1],[],[10]}$ The commonest presenting complaints were weakness $(80.1 \%)$, fever (72.1\%), dyspnea (23\%) and bleeding tendencies (11.5\%). These above findings were almost similar to the findings observed by Anita et al. ${ }^{[1]}$, Thakkar et al. ${ }^{[1]]}$ and Niazi et al..$^{[7]}$

Commonest physical sign was pallor (100\%) followed by hepatomegaly $(62.8 \%)$, splenomegaly $(47.8 \%)$ and lymphadenopathy (4.9\%). Niazi et al..$^{[7]}$ also reported pallor $(98.8 \%)$ as most common clinical sign followed by hepatomegaly $(32.5 \%)$, splenomegaly $(24.7 \%)$ and lymphadenopathy $(7.8 \%)$. Studies of other authors also showed pallor $(100 \%)$ to be the most common clinical sign. ${ }^{[1] \ll}[12]$

Analysis of Hematological Parameters: Anemia was defined as mild $(\mathrm{Hb}: 9-10 \mathrm{~g} / \mathrm{dl})$, moderate $(\mathrm{Hb}:<9 \mathrm{~g} / \mathrm{dl})$ and severe $(\mathrm{Hb}:<5 \mathrm{~g} / \mathrm{dl}){ }^{[11]}$ The mild, moderate and severe haemoglobin concentrations were $22.1 \%, 70.8 \%$ and $7.1 \%$ respectively. Thakkar et al. ${ }^{[11]}$ observed mild, moderate and severe haemoglobin concentrations as $25 \%, 51 \%$ and $24 \%$ respectively.

Leucopenia was defined as mild $(4000-2000 / \mathrm{cmm})$, moderate $(<2000-1000 / \mathrm{cmm})$ and severe $(<1000 / \mathrm{cmm}){ }^{[11]}$ Mild leucopenia was seen in $69.9 \%$ of cases, moderate in $27 \%$ and severe in $3.1 \%$. Thakkar et al. ${ }^{[1]}$ observed mild, moderate and severe leucopenia in $46 \%, 52 \%$ and $2 \%$ respectively.

Thrombocytopenia was defined as mild (1lakh-50000/ $\mathrm{cmm})$, moderate $(<50000-20000 / \mathrm{cmm})$ and severe $(<20000 /$ $\mathrm{cmm}) .{ }^{[11]}$ The platelet count was mild in $56.2 \%$ of cases, moderate in $32.3 \%$ and severe in $11.5 \%$. Thakkar et al. ${ }^{[1]]}$ 
observed mild, moderate and severe thrombocytopenia in $66 \%, 26 \%$ and $8 \%$ respectively.

We observed moderate anemia (70.8\%), mild leucopenia (69.9\%) and mild thrombocytopenia (56.2\%). Dahake et al. ${ }^{19}$ observed $39 \%$ of severe anemia, $29 \%$ of mild leucopenia and $34 \%$ mild thrombocytopenia.

The mean corpuscular volume (MCV) was increased in 65 cases, decreased in 53 cases and normal in 108 cases. Ishtiaq et al. ${ }^{[13]}$ and Soma et al. ${ }^{[5]}$ noted raised MCV in 37 cases and 13 cases respectively. The diagnosis of megaloblastic anemia should be considered when MCV is above 100fL. ${ }^{[13]}$

The predominant blood picture was normocytic normochromic (38.5\%) followed by dimorphic (32.7\%), macrocytic (17.3\%), microcytic hypochromic $(5.8 \%)$ and normocytic hypochromic $(5.8 \%)$. A study by Sweta et al. ${ }^{[14]}$ showed macrocytic anemia $(49 \%)$ where as Khodke et al. ${ }^{[15]}$ and Gayathri et al. ${ }^{[16]}$ showed dimorphic picture as the predominant blood picture.

Hypersegmented neutrophils were seen in 40 cases of megaloblastic anemia and one case of iron deficiency anemia. The studies done by Tilak et al. ${ }^{[17]}$, Gayathri et al. ${ }^{[16]}$ and Khodke et al. ${ }^{[15]}$ showed hypersegmented neutrophils in $45,43 \& 20$ cases respectively. We observed, anisopoikilocytosis in 104 cases. The studies by Tilak et al. ${ }^{[17]}$, Khunger et. ${ }^{[8]}$, Gayathri et al. ${ }^{[16]}$ and Khodke et al. ${ }^{[15]}$ showed anisopoikilocytosis in $64,150,90$ $\& 30$ cases respectively. Nucleated red blood cells were seen in 12 cases. Gayathri et al. ${ }^{[16]}$ and Khodke et a1. ${ }^{[15]}$ showed nucleated red cells in 1 \& 4 cases respectively. Reactive lymphocytes were seen in dengue (30 cases), tuberculosis (3cases), AIDS and hypersplenism (2 cases each) and septicaemia (1 case). Relative lymphocytosis was seen in dengue (41 cases) and malaria (8 cases). The studies done by Tilak et al. ${ }^{[17]}$, Khunger et al. ${ }^{[8]}$, Gayathri et al. ${ }^{[16]}$ and Khodke et al. ${ }^{[15]}$ showed relative lymphocytosis in $11,38,16 \& 5$ cases respectively. Bone marrow were hypercellular in 13 cases ( 8 cases: megaloblastic anemia, 2 cases : iron deficiency anemia and dimorphic anemia and one case: MDS) whereas 3 cases of aplastic anemia and 1 case of dyskeratosis congenita showed hypocellular bone marrow.

Causes of pancytopenia: The causes of pancytopenia in order of decreasing frequency were malaria $(50 \%)$, megaloblastic anemia (18.6\%), dengue (18.1\%), hypersplenism $(7.1 \%)$, iron deficiency anemia $(2.7 \%)$, septicemia (2.2\%), AIDS (1.8\%), tuberculosis (1.8\%), aplastic anemia (1.3\%), leptospirosis $(0.9 \%)$, dimorphic anemia $(0.9 \%)$, dyskeratosis congenita $(0.4 \%)$ and myelodysplastic syndrome $(0.4 \%) . \quad 4$ cases $\quad(0.4 \%)$ remained undiagnosed.

Malaria was the commonest cause of pancytopenia which was consistent with the finding of Hamid et al. ${ }^{[12]}$ and Tareen et al. ${ }^{[18]}$. Studies done by Parmar et al. ${ }^{[19]}$ and Thakkar et al. ${ }^{[11]}$ showed malaria as second most common cause of pancytopenia. Out of 113 cases, 65 were males and 48 females. Tareen et al. ${ }^{[18]}$ in their study also showed male preponderance with 34 males and 19 females. Maximum cases were seen in the age group of 15-25 years ( 45 cases) followed by $26-35$ years ( 26 cases) and 36-45 years (14 cases). Hamid et al. ${ }^{[12]}$ and Tareen et al. ${ }^{[18]}$ observed 1630 years and $>40$ years of age group being commonly affected by malaria.

On peripheral smear examination, there were 65 cases of P. vivax, 31 cases of mixed malaria and 17 cases of $P$. falciparum. Pancytopenia can occur in P. vivax infection secondary to microangiopathic hemolytic anemia. A report from India revaled that pancytopenia is a atypical manifestation of P. vivax which occurs in only $0.9 \% \cdot{ }^{[20]}$ It has been recently reported that both non-immunological destruction and immune mechanism involving specific platelet-associated IgG antibodies that bind directly to malarial antigen in the platelets can lead to lysis of platelets. ${ }^{[21]}$

Malaria cases showed $\mathrm{Hb}$ in the range of $3.7-9.9 \mathrm{~g} / \mathrm{dL}$, TLC: $700-3980 / \mathrm{cmm}$ and platelet count: $12,000-98,000 /$ $\mathrm{cmm}$. Both thick and thin smears showed presence of ring forms, trophozoites, mature schizonts of P. vivax and in P. falciparum ring forms and gametocytes were seen. In mixed malaria, combined features of $\mathrm{P}$. falciparum and $\mathrm{P}$. vivax were seen.

P. falciparum malaria may cause pancytopenia as a result of direct bone marrow invasion by a parasite, immune hemolysis, DIC, hypersplenism, bone marrow necrosis or hemophagocytosis. ${ }^{[22]}$ Rapid malarial antigen (RMA) test showed positivity for same types of malaria parasite as seen on peripheral smear. Apart from geographic heterogeneity, seasonal variations also influence the prevalence of malaria. The increase is particularly seen in P. falciparum. ${ }^{[23]}$

Megaloblastic anemia (18.6\%) was the second common cause of pancytopenia which coincides with studies done by Niazi et al. ${ }^{[7]}(24.7 \%)$ and Soma et al..$^{[5]}(21.7 \%)$. In various studies, the incidence varied from $3.6 \%$ to $74 \%$. The variation of incidence of megaloblastic anemia depends on the status of the nutritional anemia in that particular region of the study ${ }^{[24]}$ All cases in our study showed low levels of serum vitamin B12. 
The commonest age group affected was 15-25 years, which was consistent with the findings observed by Anita et al. ${ }^{[1]}$ In present study, males $(78.6 \%)$ were affected more than females $(21.4 \%)$, the male to female ratio being $3.66: 1$ which is comparable to the ratio $(2.7: 1)$ noted by Sweta et al. ${ }^{[14]}$ We observed weakness $(95.2 \%)$, fever (38.1\%) and dyspnea $(33.3 \%)$ as most common presenting complaints. Sweta et al. ${ }^{[14]}$ observed dyspnea (54\%) and fever (43\%) as common presenting complaints. In this study, no case presented with bleeding tendencies. Memon et al. ${ }^{[25]}$ observed pallor with varying degree of skin and mucosal bleedings in megaloblastic anemia. The most common clinical sign was pallor ( $97.6 \%$ ) followed by hepatomegaly $(23.8 \%)$, splenomegaly $(21.4 \%)$ and lymphadenopathy $(2.4 \%)$ which was similar to the findings of Khunger et al. ${ }^{[8]}$

Megaloblastic anemia cases showed haemoglobin in the range of 2.9 to $9.6 \mathrm{gm} / \mathrm{dL}$, TLC: 1300 to $3990 \mathrm{cells} / \mathrm{cmm}$ and platelet count: 8,000 to $94,000 / \mathrm{cmm}$. Amongst 42 cases, 40 cases showed increased MCV and 2 cases normal $\mathrm{MCV}$.

Peripheral smear showed macroovalocytes, anisopoikilocytosis (100\%), nucleated red cells (7.1\%) hypersegmented neutrophils $(95.2 \%)$, basophilic stippling (35.7\%) and Howell-Jolly bodies (19\%). Bone marrow was performed in 8 cases which were hypercellular showing erythroid hyperplasia. Megaloblasts, giant metamyelocytes, band forms, howell-Jolly bodies, basophilic stippling and large hyperlobated megakaryocytes were also seen.

Dengue (18.1\%) was the third common cause of pancytopenia with 24 cases of males and 17 cases of females showing male to female ratio of $1.4: 1$. Ghosh et al. ${ }^{[26]}$ also showed male preponderance with male to female ratio of $2.1: 1$. The incidence in other studies was low and varied from $0.9 \%$ to $4 \%$ which is in sharp contrast to our study. The most common age group affected was 26-35 years followed by 36-45 years and 15-25 years. The age group affected in the studies done by Santra et al. ${ }^{[22]}$ and Ghosh et al. ${ }^{[26]}$ were $13-30$ years and 21-30 years respectively. The most common presenting complaints were weakness (92.7\%), fever (90.2\%), dyspnea (24.4\%) and bleeding tendencies (19.5\%). The most common clinical sign was pallor (100\%) followed by hepatomegaly $(80.5 \%)$ and splenomegaly $(22 \%)$. Study done by Ghosh et al. ${ }^{[26]}$ reported fever as the commonest presenting complaint along with rashes, joint pains and myalgia.

Dengue cases showed $\mathrm{Hb}$ in the range of 3.1-9.9 $\mathrm{g} / \mathrm{dL}$, TLC: $0.6-3.84$ cells/cmm and platelet count: 4000-98,000/ $\mathrm{cmm}$. Anisopoikilocytosis was seen in 26 cases, reactive lymphocytes in 30 cases and relative lymphocytosis was seen in all cases. Azin et al. ${ }^{[28]}$ also noted lymphocytosis as a common finding with presence of atypical lymphocytes, bleeding and transient bone marrow suppression. Out of 41 cases, 6 cases also had megaloblastic anemia. All cases were reactive on rapid solid phase immunochromatographic card test. The epidemiology of dengue fever in the Indian subcontinent has been very complex and has changed over almost past six decades in terms of prevalent strains, affected geographical locations and severity of disease. [4] The Maharashtra State, as a whole, saw a $47 \%$ rise in dengue cases in 2012 with second highest number of deaths after Tamil Nadu. The incidence of dengue was found to be $17.95 \%$ in Kamothe, Navi Mumbai. ${ }^{[5]}$

Malaria and dengue fever are both endemic in India with active transmission being reported for many years. Thus, there is a possibility of co-existing malaria and dengue infection in the same patient. ${ }^{[28]} 4$ cases of dengue were simultaneously positive for P. vivax (2 cases), P. falciparum (1case) and mixed malaria (1case). Coinfection can be more severe than single infection with severe thrombocytopenia and anemia. Failure to recognise malaria and dengue co-infection would delay proper treatment and result in increase morbidity and mortality. ${ }^{[28]}$

Hypersplenism (7.1\%) was the fourth common cause of pancytopenia. The incidence in other studies varied from $0.9 \%$ to $28 \%$. In studies done by Jain et al. ${ }^{[10]}$, Hamid et al. ${ }^{[12]}$ and Thakkar et al. ${ }^{[11]}$ it was first, second and third common cause of pancytopenia respectively.

Iron deficiency anemia (2.7\%) was the fifth common cause of pancytopenia. In other studies, the incidence ranged from $1.3 \%$ to $13 \%$. Anita et al. ${ }^{[1]}$ and Ishtiaq et al. ${ }^{[13]}$ showed IDA as the second and fourth common cause of pancytopenia respectively. Iron studies were done in all cases for confirmation. Peripheral smear examination revealed microcytic hypochromic blood picture. Bone marrow aspiration showed hypercellular marrow with altered M:E ratio, increased erythropoiesis showing micronormoblasts. Myelopoiesis and megakaryopoiesis were normal.

In present study, septicemia accounted for $2.2 \%$ whereas in other studies varied from $2.5 \%$ to $11.3 \%$. Blood cultures were performed in all the cases to find out the causative organisms.

HIV infection was an uncommon cause with an incidence of $1.8 \%$. In other studies, the incidence varied from $1 \%$ to $12 \%$. Savage et al ${ }^{[29]}$ observed AIDS as the fourth common cause of pancytopenia. ${ }^{[29]}$ HIV infection and overwhelming bacterial infections are known to cause various hematological manifestations including pancytopenia. ${ }^{[10]}$ The hematological abnormalities may be the first clinico- 
hematological manifestations of HIV infection and AIDS involving all cell lineages of blood.

Tuberculosis is a common major public health problem in developing countries like India. Tuberculosis $(1.8 \%)$ was an uncommon cause in our study. The incidence in other studies ranged from $0.5 \%$ to $16.7 \%$. Tareen et al. ${ }^{[18]}$ reported tuberculosis as the third common cause of pancytopenia. Cases with unexplained pyrexia, weight loss and pancytopenia should be investigated for Tuberculosis. ${ }^{[10]}$ We show 3 cases of Pulmonary tuberculosis and one case of disseminated tuberculosis. Disseminated tuberculosis is known to cause pancytopenia but there are reports of pulmonary tuberculosis too causing pancytopenia. ${ }^{[10]}$ The studies conducted by Ishtiaq et al. ${ }^{[13]}$, Tilak et al..$^{[17]}$ and Santra et al. ${ }^{[22]}$ showed an incidence of $3 \%, 0.5 \%$ and $0.9 \%$ of disseminated tuberculosis respectively.

Aplastic anemia contributed to $1.3 \%$ which is in contrast to the findings observed by other authors where aplastic anemia was the most common cause of pancytopenia. Few studies have also reported low incidence of aplastic anemia. ${ }^{[1] \&[24]}$ Bone marrow aspiration showed hypoplastic marrow.

Leptospirosis was a rare cause of pancytopenia with an incidence of $0.9 \%$ and has been scarcely mentioned in the literature. They presented with fever, weakness, bleeding tendencies and pallor. The diagnosis of leptospirosis was made by immunochromatographic card test.

Dimorphic anemia showed a low incidence $(0.9 \%)$ as compared to other studies where it ranged from $1.88 \%$ to $20 \%$. All cases showed low levels of serum B12 and serum ferritin. Bone marrow were hyperceullar with micronormoblastic and megaloblastic erythropoiesis.

Myelodysplastic syndrome was one of the least common causes of pancytopenia accounting for $0.4 \%$. The incidence in other studies ranged from $0.4 \%$ to $5.6 \%$. Ishtiaq et al. ${ }^{[13]}$ showed an incidence of 5\%.

Dyskeratosis congenita (DC) was a very rare cause of pancytopenia with an incidence of $0.4 \%$. This is in contrast to the studies reviewed in literature, wherein no case of DC causing pancytopenia has been mentioned. However, there are few case reports on DC. We encountered a case of 20year old male, resident of Maharashtra came with fever, weight loss, weakness and dyspnea with no significant family history. He presented with pallor, reticular hypopigmentation and hyperpigmentation of the skin, dystrophic nails, graying of hair and oral leukoplakia. There was severe anemia with haemoglobin of $4.2 \mathrm{~g} / \mathrm{dl}$, TLC of $1150 / \mathrm{cmm}$ and platelet count of $24000 / \mathrm{cmm}$. Bone marrow aspiration revealed a hypoplastic picture with reduction in all three cell lineages and relative increase in plasma cells and few lymphocytes. Genetic studies showed confirmation of mutation in DKC1 gene.

\section{Conclusion}

Pancytopenia is a common haematological problem encountered in clinical practice. Malaria was the most common cause of pancytopenia followed by megaloblastic anemia and dengue. The less common causes included hypersplenism, iron deficiency anemia, septicemia, AIDS, tuberculosis, aplastic anemia, MDS, dimorphic anemia, leptospirosis and dyskeratosis congenita. Detailed clinical history and meticulous physical examination along with baseline haematological investigations helps in early diagnosis and thus avoiding battery of unnecessary investigations while evaluating a pancytopenic patient.

\section{Acknowledgements}

I would like to acknowledge the entire technical staff for providing sincere assistance in this study and contributing for the same.

\section{References}

1. Javalgi AP, Dombale VD. Clinico-hematological analysis of pancytopenia: A bone marrow study. National Journal of Laboratory Medicine 2013, 2(4):12-17.

2. Aziz T, Ali L, Ansari T, Liaquat HB, Shah S, Ara J. Pancytopenia: Megaloblastic anemia is still the commonest cause. Pak J Med Sci. 2010; 26:132-136.

3. Lakhey A, Talwar OP, Singh VK, KC Shiva Raj. Clinicohematological study of pancytopenia. Journal of Pathology of Nepal. 2012; 2:207-210.

4. Tonape SD, Shende NY. To study Hemogram and Bone Marrow Morphology (Aspiration/ Biopdy) in cases of Pancytopenia at GMC \& H Nagpur- Two Year Observational Study. Translational Medicine and Biotechnology. 2014; 2(3):15-26.

5. Yadav S, Kushwaha R, Aggrawal K, Tripathi AK, Singh US, Kumar A. A Clinico- Hematological Study in Cases of Pancytopenia: Correlation of Automated Cell Counter Parameters in Various Etiologies. Journal of Evolution of Medical and Dental Sciences. June 3, 2013; 2(22):40134023.

6. Naseem S, Varma N, Das R, Ahluwalia J, Singh MU, Sachdeva, Marwaha RK. Paediatric patients with bicytopenia/ pancytopenia: Review of etiologies and clinicohematological profile at a tertiary center. Indian $\mathrm{j}$ Pathol Microbiol 2011; 54: 75-80.

7. Niazi M, Raziq F. The incidence of underlying pathology in Pancytopenia- An experience of 89 cases. JPMI, 2004; 18(1): 76-79.

8. Khunger JM, Arulselvi S, Sharma U, Ranga S, Talib VH. Pancytopenia- A Clinico-hematological study of 200 cases. Indian J. Pathol. Microbiol. July, 2002; 45(3): 375-379. 
9. Dahake V, Margam S, Gadgil N, Patil M, Kalgutkar A. Clinico-hematological Analysis of Pancytopenia in Tertiary Care Hospital. International Journal of Scientific Study. November 2014; 2(8): 59-63.

10. Jain A, Naniwadekar M. An etiological reappraisal of pancytopenia - largest series reported to date from a single tertiary care teaching hospital. BMC Hematology 2013; $13: 10$.

11. Thakkar BB, Bhavsar UN, Trivedi NJ, Agnihotri AS. A Study of Pancytopenia in Adult Patients more than 12 years of age in North West region of Saurashtra. 2013; 3(1): 48-52.

12. Hamid GA, Shukry SAR. Patterns of pancytopenia in Yemen. Turk J Hematol 2008; 25: 71-4.

13. Ishtiaq O, Baqai HZ, Anwer F, Hussian N. Patterns of Pancytopenia patients in a general medical ward and a proposed diagnostic approach. J Ayub Med Coll Abottabad 2004; 16(1): 8-13.

14. Sweta, Barik S, Chandoke RK. and Verma AK. A Prospective Clinico-Hematological Study in 100 Cases of Pancytopenia in Capital City of India. Journal of Applied Hematology. 2014; 5:45-50.

15. Khodke K, Marwah S, Buxi G, Yadav RB, Chaturvedi NK. Bone marrow examination in cases of pancytopenia. $\mathrm{J}$ Academy Clin Med. 2001; 2: 55-59.

16. Gayathri BN, Rao KS. Pancytopenia: A clinicohematological study. J Lab Physicians 2011; 3: 15-20.

17. Tilak V, Jain R. Pancytopenia - A Clinico-hematologic analysis of 77 cases. Indian J. Pathol. Microbiol. 1999; 42(4): 399-404.

18. Tareen SM, Tariq MM, Bajwa MA, Awan MA, Ahmad Z, Javed Y. Study of Pancytopenia in Balochistan, Pakistan. Gomal J Med Sci 2012; 10: 248-251.

19. Parmar JK, Sheikh S, Vidja P. Etiological Evaluation of Pancytopenia with Special Emphasis on Megaloblastic Anemia. Indian Journal of Research, May 2013; 3(4): 263-264.
20. Albaker W. Acute Plasmodium Vivax Malaria presenting with Pancytopenia secondary to Hemophagocytic Syndrome: Case Report and Literature Review. J Family Community Med. 2009 May-Aug; 16(2): 71-73.

21. Bhalara SK, Shah S, Goswami H, Gonsai RN. Clinical and etiological profile of thrombocytopenia in adults: A tertiarycare hospital-based cross-sectional study. Int $\mathrm{J}$ Med Sci Public Health 2015; 4: 7-10.

22. Santra G, Das BK. A cross-sectional study of the clinical profile and aetiological spectrum of pancytopenia in a tertiary care centre. Singapore Med J 2010; 51(10): 806-812.

23. Tapaswi A. Seasonal variation in the Incidence of Malaria in Navi Mumbai. Pediatric Infectious Disease, Oct-Dec 2010; 2:188-189.

24. Graham S, Marla NJ, Fernandes H, Jayaprakash CS. A clinicohematological evaluation of pancytopenia in a tertiary care hospital in South India. Muller J Med Sci Res 2015; 6: 5-9.

25. Memon S. Shaikh S, Nizamani MA. Etiological spectrum of pancytopenia based on bone marrow examination in children. J COll Physicians Surg Park 2008 Mar; 18(3): 163-7.

26. Ghosh G, Urhekar AD, Kosta S. A clinico-microbiological study of Dengue fever cases in a tertiary care centre of Navi Mumbai. Int J Bioassays, 2013; 2(11): 1462-1467.

27. Azin FRFG, Goncalves RP, Pitombeira MHDS, Lima DM, Branco IC. Dengue: Profile of hematological and biochemical dynamics. Rev Bras Hematol Hemoter. 2012; 34(1): 36-41.

28. Khan A, Aqeel M, Khan TA, Munir A. Pattern of Hematological Diseases in Hospitalized Paediatric Patients Based on Bone Marrow Examination. 2008; 22(3): 196-200.

29. Savage DG, Allen RH, Gangaidzo IT, Levy LM, Gwanzura C, Moyo A, Mudenge B, Kiire C, Mukiibi J, Stabler SP, Lindenbaum J. Pancytopenia in Zimbabwe. Am J Med Sci. 1999 Jan; 317(1): 22-32.

30. Ramzan M, Yadav SP, Sachdeva A. Post-dengue fever severe aplastic anemia: a rare association. Hematol Oncol Stem Cell Ther 2012; 5(2): 122-124.

*Corresponding author:

Dr Manisha Tambekar, Department of Pathology, MGM Institute of Health Sciences, Mumbai, Maharashtra, India

Phone: +91 919769416262, 410210

Email: manisha.tambekar@gmail.com

Financial or other Competing Interests: None. 